\title{
Erratum: Asymptotic flux compactifications and the swampland
}

\author{
Thomas W. Grimm, ${ }^{a}$ Chongchuo $\mathbf{L i}^{a}$ and Irene Valenzuela ${ }^{b}$ \\ ${ }^{a}$ Institute for Theoretical Physics, \\ Utrecht University, Princetonplein 5, 3584 CE Utrecht, The Netherlands \\ ${ }^{b}$ Jefferson Physical Laboratory, Harvard University, \\ Cambridge, MA 02138, U.S.A. \\ E-mail: t.w.grimm@uu.nl, c.li@uu.nl, ivalenzuela@g.harvard.edu
}

ERRATUM TO: JHEP06(2020)009

ARXIV EPRINT: 1910.09549

In the original paper a wrong affiliation has been assigned to author Chongchuo Li during the typesetting.

The correct affiliation for author Chongchuo $\mathrm{Li}$ is:

a Institute for Theoretical Physics, Utrecht University, Princetonplein 5, 3584 CE Utrecht, The Netherlands

Open Access. This article is distributed under the terms of the Creative Commons Attribution License (CC-BY 4.0), which permits any use, distribution and reproduction in any medium, provided the original author(s) and source are credited. 